\title{
Die Entwicklung schulisch-standardsprachlicher Kompetenzen in der Volksschule. Eine Quasi-Längsschnittstudie
}

\author{
Romano Müller/Nora Dittmann-Domenichini (Bern)
}

\begin{abstract}
The article presents the theoretical and methodological reflexions guiding the research project "The Development of Cognitive-Academic Language Proficiency of Monolingual and Bilingual Primary and Secondary School Students. A comparison" (2006-2010).

(1) The first part gives an overview of immigrant pupils' situations in the Swiss educational system and shows the reasons for their unsatisfying success in school. Swiss school system is mainly defined by an assimilative philosophy not sufficiently taking into account the specific conditions of the $23.7 \%$ of bilingual students in Swiss schools.

(2) The theoretical bases of the study refer to the framework of socio-cognitive theory. On the individual level, factors such as the "linguistic self-concept" and "intelligence", and on the exogenous level, context factors such as the "institutional support", specific "classroom programs" for migrant pupils and the "aspirations and support opportunities of the families" are supposed to have a systematic impact on the pace and the level of second language acquisition. We further discuss the theoretical conception of language competencies underlying the project.

(3) Finally the methodology of the project, including 1200 monolinguals and bilingual students, is discussed. The project follows the rules of a quasi-longitudinal study measuring the development of language competencies levels twice in the interval of one year in the $2^{\text {nd }} / 3^{\text {rd }}-, 5^{\text {th }} / 6^{\text {th }}$, and $8^{\text {th }} / 9^{\text {th }}$-grades.

The results will lead to insights in the acquisition process and eventual stagnations in educational language development.
\end{abstract}

\section{$1 \quad$ Einleitung}

Das Forschungsprojekt "Sprachliche Kompetenzen bei ein- und mehrsprachigen SchülerInnen" der Pädagogischen Hochschule Bern beschäftigt sich mit der Frage des Einflusses schulischer und familiärer Kontexte auf die Entwicklung der schulsprachlichen Kompetenzen bei mehrsprachigen SchülerInnen, im Vergleich zu ihren einsprachigen KameradInnen. Im Mittelpunkt steht dabei die Hypothese, dass die schulsprachlichen Kompetenzunterschiede zwischen den ein- und mehrsprachigen SchülerInnen durch schulische und familiäre Kontextbedingungen verursacht bzw. akzentuiert werden, und dies bei gleichzeitiger Kontrolle soziogener Variablen wie sozialer Status, Bildungsnähe, Gender, Einwanderungszeitpunkt und Begabung. Der folgende Artikel gibt zunächst einen Überblick über den aktuellen Forschungsstand zur Situation mehrsprachiger MigrantenschülerInnen im Schweizer Bildungssystem. Danach werden das sozio-kognitive Grundlagenmodell (Kap. 3 und 4) und das Sprachmodell (Kap. 5) der Untersuchung dargestellt. Schließlich werden die 
Forschungsfragen (Kap. 6), die Methoden (Kap. 7) und die Bedeutsamkeit (Kap.8) der Untersuchung dargestellt.

\section{Schulischer Erfolg und Misserfolg von mehrsprachigen SchülerInnen}

In der heutigen Gesellschaft ist Mehrsprachigkeit eine Realität, der sich alle Bildungsinstitutionen stellen müssen. Für den aussen stehenden Betrachter erscheint dabei die sprachliche Situation in der multilingualen Schweiz als Idealfall sprachlichen und kulturellen Zusammenlebens. Bezüglich der in den letzten 20-30 Jahren stetig steigenden Zahl von Kindern aus Migrationsfamilien ist der Integrationserfolg des Schweizer Bildungssystems dennoch ernüchternd. So zeigt sich in allen neueren Darstellungen der schweizerischen Migrationsforschung eine Stagnation der Erfolgsquote ausländischer bzw. mehrsprachiger SchülerInnen im schweizerischen Schulsystem mit Tendenzen zur Verschlechterung (Häberlin, Imdorf/Kronig 2004; Imdorf 2001; Kronig, Haeberlin/Eckhard 2000; Moser/Rhyn 2000; Müller 1993; 2001a; Rüesch 1998):

(1) Der Anteil ausländischer Kinder in sonderpädagogischen Zügen ist relativ betrachtet ca. viermal höher als jener der Schweizer Kinder und hat sich in den letzten 15 bis 20 Jahren mehr als verdoppelt (Kronig et al. 2000; Müller 1996; 2001b).

(2) Die Aufnahmequote in die Sekundarstufe-I-Typen mit erweiterten Ansprüchen beträgt bei den monolingualen Schweizer Jugendlichen ca. 65\%, bei den bilingualen ausländischen Jugendlichen nur ca. 35 bis 40\% (Kronig et al. 2000; BfS 1997; Müller 2001).

(3) Der Zugang zu den gymnasialen Zügen beträgt bei den SchweizerInnen ca. $21 \%$, bei den AusländerInnen ca. $10 \%$.

(4) Die Bildungsbeteiligung der ausländischen Jugendlichen in Berufslehren ist mit 38\% signifikant niedriger als bei den SchweizerInnen mit 52\%, und sie fällt besonders niedrig aus bei Angehörigen aus der Türkei, dem ehemaligen Jugoslawien, Albanien und Portugal (Imdorf 2004; Meyer 2004; Meyer, Stalder/Matter 2003; Müller 2001; $\left.2006^{\mathrm{a}} ; 2006^{\mathrm{b}}\right)$.

(5) Unter den gleichen bildungsmäßigen Voraussetzungen sind die Chancen auf einen Übertritt in die Sekundarstufe bei mehrsprachigen SchülerInnen um 25\% niedriger als bei den einsprachigen Schweizer KollegInnen (Müller 2001; 2005 ${ }^{\mathrm{b}}$ ).

\subsection{Ursächliche Faktoren des schulischen Misserfolgs}

Auch in der Interpretation dieses Sachverhaltes sind sich die meisten wissenschaftlichen AutorInnen in der Schweiz weitgehend einig: Der schulische Erfolg bzw. Misserfolg hängt in sehr hohem Masse von den, im Verlauf einer (vor-)schulischen Karriere erworbenen, schulsprachlichen (standardsprachlichen) Kompetenzen ab.

Zentral bei Selektionsentscheiden ist in erster Linie das Erreichen von definierten schulischsprachlichen Standards oder allgemeiner: der schulsprachlich definierten Kompetenzen. Dies heisst nicht, dass bei einem Teil der ausländischen mehrsprachigen SchülerInnen nicht andere Leistungsschwächen vorhanden wären. Jedoch zeigen Analysen, dass auch unter der Kontrolle anderer schulischer Selektionsfächer (Mathematik und Fremdsprache) und nichtschulischer Leistungen (IQ-Werte) das schulische Versagen hauptsächlich mit geringeren L2-sprachlichen Leistungen zusammenfällt (Imdorf 2004; Kronig 2003; Kronig et al. 2000; Müller 1997; 2000).

Insbesondere lässt sich die Varianz bei den Deutschleistungen nicht auf die soziale Herkunft reduzieren, wie dies in der schweizerischen Migrationspädagogik allenthalben behauptet wird 
(Corradi Vellacott/Wolter 2002; Hutmacher 1995). Wenn die Faktoren der sozialen Schichtzugehörigkeit und der Bildungsnähe beim Entstehen der Varianz des schulischen (und spezifisch des schulsprachlichen) Erfolgs mitwirken, so tragen sie in keiner der systematisch kontrollierten schweizerischen Untersuchungen das Hauptgewicht des schulischen Erfolgs bzw. Misserfolgs. Es kann auch aufgezeigt werden, dass bezüglich der schulsprachlichen Leistungen (gemessen im Lehrerurteil und durch objektivierte Testverfahren) die soziale Schichtung im Unterschied zur sprachlichen Herkunft keine signifikanten Effekte erzeugt ${ }^{1}$ (Müller 1997; 2006 ; 2006 ${ }^{\text {) }}$.

Die Dominanz der schulsprachlichen Beherrschung bei Selektionsprozessen wurde in der Schweiz bisher allerdings nicht direkt aufgezeigt. Jedoch lässt sich diese Dominanz in ihren Auswirkungen indirekt nachweisen. In den zwei niedrigeren Leistungstypen der Sekundarstufe I (Realschule und Sekundarschule) schliessen die mehrsprachigen SchülerInnen, unabhängig von der Schichtzugehörigkeit, signifikant besser im Fach Mathematik und in der Fremdsprache Französisch ab als ihre einsprachigen KollegInnen. Trotzdem befindet sich ein überdurchschnittlich hoher Anteil von mehrsprachigen SchülerInnen in der Realschule, während dies nur bei einem weit geringeren Teil der einsprachigen SchweizerInnen der Fall ist. Im Bereich der standardsprachlichen Kompetenzen sind die Leistungen der mehrsprachigen SchülerInnen auf einem signifikant niedrigerem Niveau (Müller 1997; 2000). Dieser Sachverhalt kann als Ausdruck der starken Gewichtung der standardsprachlichen Lokalsprache L2 im Selektionsprozess interpretiert werden. Im höchsten (gymnasialähnlichen) Leistungstypus der Sekundarstufe I sind die Unterschiede nicht mehr signifikant. Es lässt sich auch feststellen, dass sich die Schulen mit der Tatsache der Mehrsprachigkeit eines grossen Teils ihrer SchülerInnen schwer tun. So wird in Selektionsentscheiden die Mehrsprachigkeit der SchülerInnen, trotz gegenteiligen Empfehlungen der $\mathrm{EDK}^{2}$, in der Praxis selten berücksichtigt. Die Schulen tun sich auch schwer mit der Integration der freiwilligen HSK-Kurse ${ }^{3}$ in den normalen Stundenplan. Es fehlen zudem integrative Zweisprachigkeitsansätze in der Schule. Das schweizerische Modell entspricht im Wesentlichen einem monolingual-assimilativen Spracherziehungsansatz.

\subsection{Politisch-rechtliche Hintergründe assimilativer Einsprachigkeitsmodelle in der Schweiz}

Der monolinguale Habitus der Schweizer Schulen liegt im verfassungsrechtlich verankerten sprachlichen Territorialitätsprinzip (Art. 70 BV) begründet. Dieses sichert den Ausgleich der Landessprachen untereinander und schreibt vor, dass die jeweilige Lokalsprache als Schulsprache zu gelten hat. Dies gilt sowohl für fremdsprachige Migrantenschüler als auch für Schweizer SchülerInnen, deren Muttersprache, auch wenn sie eine der vier Schweizer Landessprachen ist, nicht dem lokalen Idiom entspricht. Das sprachliche Territorialitätsprinzip hat dazu beigetragen, den sprachlichen Frieden in der Schweiz zu sichern. Es hat aber auch bewirkt, dass in der schweizerischen Schullandschaft die Entwicklung von eigentlichen schulischen Zweisprachigkeitskonzepten (im Unterschied zu Fremdsprachenkonzepten) sehr bescheiden ist.

\footnotetext{
${ }^{1}$ Es lassen sich jedoch varianzanalytisch kreuzweise Effekte feststellen, die zeigen dass sich die sprachliche Zugehörigkeit in der unteren sozialen Schicht stärker auswirkt als in der Mittel- und Oberschicht.

2 Die EDK ist die Schweizerische Konferenz der kantonalen Erziehungsdirektorinnen und Erziehungsdirektoren.

3 HSK-Kurse sind Kurse in heimatlicher Sprache und Kultur. Sie werden meist von den Konsulaten der Herkunftsländer für Migrantenkinder angeboten, mit dem Ziel diese, überwiegend in der Familie gesprochenen Sprache, auf ein schulsprachliches Niveau zu heben.
} 


\subsection{Soziologische Hintergründe assimilativer Einsprachigkeitsmodelle in der Schweiz}

Der assimilativ-monolinguale Charakter des Schweizer Schulsystems kann jedoch nicht als ein zufälliges Produkt historischer Schulentwicklung gesehen werden. (Schulische) Auslesepraktiken spiegeln auch immer die Interessen von majoritären (monolingualen) Gruppen gegenüber kompetitiven Ansprüchen minoritärer (in diesem Falle bilingualen) Gruppen wider. Die Sprache, beziehungsweise die Selektion via schulischstandardsprachlicher Kompetenzen, eignet sich in besonderer Weise dafür, den Zugang einzelner Bevölkerungsgruppen $\mathrm{zu}$ (höheren) Bildungsgängen und damit $\mathrm{zu}$ den ökonomischen Ressourcen zu steuern. Die schulische Selektion gemäss den lokalen schulsprachlichen Standards erweist sich als eines der probaten Instrumente der Grenzziehung, das dominante soziale Gruppen gegenüber kompetitiven Minderheitsgruppen anwenden. Sie sichern sich mit diesem Ausleseverfahren die eigenen Wettbewerbschancen und damit den Zugang zu bildungsmäßigen und ökonomischen Gütern und regulieren auf diese Weise nach Bedarf die Bildungschancen der anderssprachigen Minoritätsgruppen. Vom Standpunkt der Intergruppentheorie spiegeln die genannten sozialen Regulierungsstrategien die in den industrialisierten Gesellschaften deutlich zu beobachtende Tendenz zur Favorisierung der eigenen sozialen Gruppe wider (Tajfel 1978; Tajfel/Turner 1979).

\section{Wissenschaftlicher Stand zur Zweisprachigkeitsforschung}

Angesichts der Fülle empirischer und theoretischer Studien zum Zweitsprachenerwerb ist es wichtig, das vorliegende Projekt theoretisch zu verorten.

Das Forschungsprojekt "Sprachliche Kompetenzen" interessiert sich für den Einfluss der schulischen Kontexte: "Schule-Institution", "Schule-Unterricht" und "Familiäre Aspiration und Hilfestellung sowie Beziehung Elternhaus - Schule" auf die Entwicklung der schulsprachlichen Kompetenzen. Seine theoretische Orientierung ist den sozio-kognitiven Theorieansätzen zuzuordnen. Sie beziehen sich unter anderem auf Albert Banduras Konzept der Selbstwirksamkeit als zentraler Handlungsprädiktor (Bandura 1977; 1986; 1997). Innerhalb der Zweisprachigkeitsforschung stützt sich das Projekt auf die Arbeiten der Gardner-Lambert Schule und insbesondere auf das darauf aufbauende sozio-kognitive Modell des schulischen Zweitsprachenerwerbs von Müller (1997). Letzteres wird nach einem Überblick über die Arbeiten von Gardner, Lambert und Clément etwas ausführlicher dargestellt.

Eingangs sollte erwähnt werden, dass sich die Modelle von Lambert, Gardner und Clément im Unterschied zu Müllers Modell nicht eigentlich auf den Zweitsprachenerwerb von MigrantInnen beziehen. Ihr empirisches Forschungsobjekt ist vielmehr der Fremdsprachenerwerb in mono- bzw. bikulturellen Milieus Kanadas und Nordamerikas. Dies bedingt dann auch Unterschiede in den empirischen Ergebnissen, wie in der Folge zu zeigen sein wird.

\subsection{Lamberts Modell der L2 sprachlichen Identität}

Lamberts Modell (Lambert 1974; 1979; 1981) rückt motivationale Tendenzen beim L2Erwerb in das Zentrum der Überlegungen und legt das Augenmerk auf die Identitätsentwicklung, die mit dem Zweit- bzw. Fremdsprachenerwerb einhergeht. Die Bereitschaft bzw. der Wille zur Übernahme von Werten und Lebensweisen der umgebenden Mehrheitsgesellschaft bezeichnet Lambert als integrative Orientierung. Sie ist die zentrale Variable in Lamberts Modell und Voraussetzung für erfolgreichen L2-Erwerb. Sie ist abzugrenzen von der instrumentellen Orientierung, welche im Zweitsprachenerwerb vornehmlich ein Mittel zum Erlangen ökonomischer oder praktischer Vorteile sieht. 
Die in Lamberts Modell postulierten Orientierungen und Einstellungen zur L2-Gruppe (Grad an Ethnozentrismus) wirken sowohl direkt als auch indirekt, vermittelt über die Motivation ${ }^{4}$ auf die L2-Sprachbeherrschung. Lambert zieht das ethnolinguistische Setting in seine Betrachtungen mit ein, und postuliert unterschiedliche Formen des Bilingualismus (additiver versus subtraktiver Bilingualismus), in Abhängigkeit des Verhältnisses zwischen der majoritären und minoritären Gruppe.

\subsection{Gardners sozio-erzieherisches Modell}

Das sozio-erzieherische Modell (Gardner 1979; 1985) gibt einen Einblick in den Zusammenhang sorgfältig erforschter Variablen und Teilprozesse des L2-Erwerbs. Es baut auf dem Modell von Lambert auf, differenziert jedoch zusätzlich zum soziokulturellen Milieu auch nach L2-Erwerbskontext (formell versus informell).

Gardner postuliert vier vermittelnde Variablen (Intelligenz, Sprachbegabung, Motivation, situationale Angst), welche den Grad der L2-Beherrschung beeinflussen. Die Gewichtung des Einflusses der Variablen kann je nach kulturellem Milieu unterschiedlich sein. Gardner geht davon aus, dass in monokulturellen Settings Zweitsprachenerwerb überwiegend in formellen Lernkontexten erfolgt. Er vermutet, dass die kognitiven Begabungsfaktoren vor allem in diesen formellen Lernkontexten zum Tragen kommen. Demgegenüber sind die die motivationalen Variablen sowohl in formellen als auch informellen Lernsituationen von Bedeutung. Gardner konnte empirisch nachweisen, dass (1) die Sprachbegabung für die Vorhersage des L2-Erfolgs in beiden Kontexten relevant ist und (2) ihre Bedeutung in monokulturellen Kontexten tatsächlich grösser ist als in bikulturellen Kontexten.

Gardner ergänzt Lamberts Modell unter anderem durch den Einbezug der Persönlichkeitsvariable "situationale Angst vor dem L2-Gebrauch". In bikulturellen Settings lassen sich substantielle Korrelationen zwischen der Angstvariablen und dem mündlichen L2Gebrauch feststellen (Gardner, Lalonde/Pierson 1983; Lalonde/Gardner 1984). Spätere Studien (Gardner, Moorcroft/Metforda 1989) lassen vermuten, dass die Angstvariable das Umkehrmass der Variable Selbstvertrauen ist. Beide Persönlichkeitsvariablen stehen in einem engen Zusammenhang zum L2-Erwerbserfolg.

Durch die systematische, empiriegeleitete Prüfung und Weiterentwicklung seines Modells gelingt es Gardner qualitative Aussagen über den Anteil der Motivation, in Abhängigkeit des jeweiligen soziokulturellen Kontextes, zu machen. Er zeigt, dass die Bedeutung des integrativen Motivs unter bestimmten sozialen Bedingungen schwankt und durch das Gewicht der situationalen Angst oder der instrumentellen Motivation kompensiert werden kann. Zudem wird belegt, dass Einstellungen, zur Zielsprache oder zur majoritären Umgebungskultur, erst vermittelt über die Motivationsstärke für die Vorhersage der L2Sprachbeherrschung relevant werden.

\subsection{Cléments soziales Kontextmodell}

Clément baut in seinem sozialen Kontextmodell die Differenzierung des sozialen und kulturellen Erwerbskontextes noch weiter aus. Während Gardner kritisiert wurde, ethnolinguistische Variablen wie Gruppengrenzen und Gruppenvitalität zu sehr zu vernachlässigen (Giles/Byrne 1982), versucht Clément Aspekte des sozio-erzieherischen Modells und der Intergruppentheorie in sein Modell einzubinden. Dem ethnolinguistischen Kontext wird eine grössere Bedeutung beigemessen und die wahrgenommene

\footnotetext{
${ }^{4}$ Es handelt sich in Lamberts Modell bei der Motivation um eine Anhäufung von Teilvariablen bestehend aus gruppenspezifischen Einstellungen, Einstellungen zum L2 Unterricht, Indikatoren der Motivationsstärke und allgemeinen Einstellungen (z. B. Interesse an Sprachen, Ethnozentrismus).
} 
Gruppenvitalität wird als Strukturvariable ins Modell aufgenommen. Clément übernimmt von Lambert und Gardner die integrative Motivation und die Angstkomponente als wesentliche strukturelle Variable in sein Modell. Jedoch handelt es sich nicht mehr um "situationale Angst vor L2- Gebrauch" sondern um Assimilationsangst "which corresponds to the fear that loss of the first culture and language might result from learning the second language" (Clément 1980:149). Das Verhältnis zwischen der integrativen Motivation und der Assimilationsangst bestimmt die Stärke der Motivation zum Lernen der L2 (primärer Motivationsprozess). Überwiegt die Assimilationsangst, so resultieren daraus Vermeidungsverhalten und eine geringe Motivation für den L2-Erwerb. Überwiegt jedoch die Stärke der integrativen Motivation, so erwartet Clément vom Lerner Annährungsverhalten an die L2-Gruppe und eine hohe Motivation für den L2-Erwerb. In bikulturellen Kontexten wirken zusätzlich die Kontakthäufigkeit und die Kontaktqualität und das daraus resultierende Selbstvertrauen des L2-Lerners als Strukturvariablen (sekundärer Motivationsprozess).

\subsection{Das sozio-kognitive Modell von Müller}

Müller (1997) greift in seiner Strukturstudie die drei Modelle auf und prüft die von Lambert, Gardner und Clément zusammengetragenen Faktoren in ihrem komplexen Zusammenhang mit der L2-Kompetenz. Er kommt dabei zum Schluss, dass der Erwerb der schulischkognitiven Sprache psychologisch betrachtet von nur zwei Variablen angemessen vorhergesagt werden kann:

(1) der Begabung (Sprachbegabung und nicht-sprachliche Intelligenz) und

(2) dem schulsprachlichen Selbstkonzept (oder schulsprachliches Selbstvertrauen).

Dies trifft sowohl für die Gruppe der einsprachigen als auch der mehrsprachigen SchülerInnen zu. Dadurch wird nachgewiesen, dass es sozialpsychologisch keine strukturellen Unterschiede zwischen mehrsprachigen und einsprachigen SchülerInnen gibt ${ }^{5}$. Der Einfluss der Begabungsfaktoren und der motivationalen Komponente wird bestätigt. Entgegen der Annahme von Gardner, Lambert oder Clément, spielen die sozio-affektiven Variablen ${ }^{6}$ und die Variablen des Ausmasses der Integration ${ }^{7}$ bei Mehrsprachigen nur eine untergeordnete Rolle für die Voraussage der schulsprachlichen Kompetenz. Die entscheidende sozialpsychologische Variable ist das Vertrauen in die eigenen schulsprachlichen Fähigkeiten und das daraus resultierende sprachliche Selbstkonzept. Es ist das Ergebnis einer langen Ansammlung von Erfahrungen und Rückmeldungen bezüglich des eigenen L2-sprachlichen Leistungsvermögens. Im Ergebnis entsteht eine präzise Beurteilung der eigenen L2sprachlichen Fähigkeiten, welche die tatsächlich erzielten Ergebnisse besser vorhersagt als die allgemeine Leistungsmotivation oder die Einstellung zum Lernen und zur Schule.

Die Wirkung der dynamischen Aspekte der Motivation wird von Müller nicht grundsätzlich in Frage gestellt, sie wird aber nuanciert.Die Motivationsstärke ist nur dann ein guter Prädiktor für die schulsprachlichen Leistungen, wenn gleichzeitig ein hohes Selbstvertrauen vorhanden ist, welches die Motivation auf ein präzises Ziel hin orientiert. Ebenso wie der Einfluss der

\footnotetext{
${ }^{5}$ Es gibt aber graduelle Unterschiede in der Ausprägung der einzelner Variablen. Müller kommt zu dem Schluss, dass der Einfluss der Intelligenz bei mehrsprachigen SchülerInnen auf den schulsprachlichen Erfolg grösser ist als bei monolingualen SchülerInnen. Die strukturellen Gegebenheiten des Modells gelten jedoch für beide Gruppen.

6 Es handelt sich dabei um die Variablen "soziales schulisches Selbstkonzept / Einstellung zu KameradInnen, LehrerInnen und Schulklima", bei Clément werden diese als Variablen des sekundären Motivationsprozess bezeichnet.

7 Gemeint sind die Variablen "sprachliche Integration", "soziale Integration" und "Zahl und Qualität der Kontakte zu einsprachigen SchweizerInnen".
} 
motivationalen Stütze zur Verbesserung der schulsprachlichen Leistungen ist auch die Wirkung von kurzfristigen didaktischen Massnahmen und Ermunterungen äusserst limitiert.

Die unterschiedlichen Ergebnisse hinsichtlich des Einflusses der motivationalen Variablen und der Integrationsvariablen zwischen Müller und Lambert, Gardner, Clément erklären sich auch durch Unterschiede in der untersuchten Stichprobe. Während bei Lambert, Gardner und Clément kanadische und nordamerikanische SchülerInnen oder StudentInnen beim Erlernen einer Fremdsprache (Englisch oder Französisch) untersucht wurden, handelt es sich bei Müller um MigrantenschülerInnen, die seit vielen Jahren in der Schweiz leben. Ihre Integration ist nicht mehr abhängig von ihrer Motivation zur Kontaktaufnahme und Integration, da sie bereits erfolgt ist. Es geht somit bei Müller um den Zweitsprachenerwerb von integrierten MigrantenschülerInnen.

\section{$4 \quad$ Das theoretische Modell der Untersuchung}

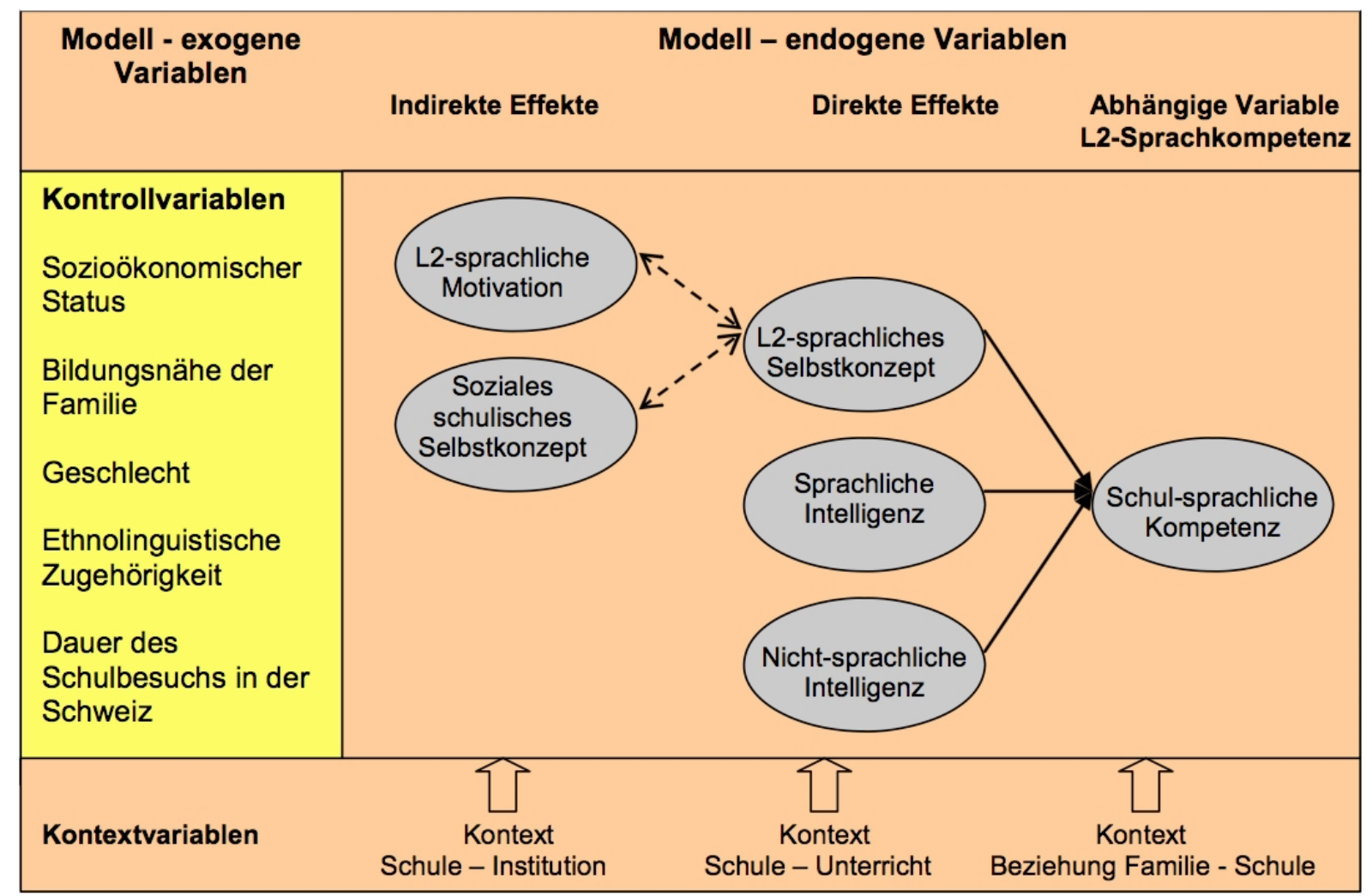

Abb. 1: Das sozio-kognitive Modell nach Müller (1997)

In der vorliegenden Studie, wird das Modell von Müller aufgegriffen und hinsichtlich der abhängigen Variable weiter differenziert (Abb.1). Als modell-exogene Variablen fungieren zum einen die Kontrollvariablen (sozioökonomischer Status, Geschlecht, ethnolinguistische Zugehörigkeit, Dauer des Schulbesuchs in der Schweiz, Bildungsnähe der Familie) und zum anderen die Kontextvariablen. Letztere beziehen sich auf die Einstellung und Merkmale der schulischen Institution im Hinblick auf die Förderung Mehrsprachiger, auf Qualitätsmerkmale des Unterrichts im Hinblick auf die Förderung Mehrsprachiger sowie auf die Beziehung zwischen Elternhaus und Schule.

Während bei Müller die L2-Sprachkompetenz über das LehrerInnen-Urteil bzw. die Leistung der SchülerInnen im Allgemeinen Deutschen Sprachtest (Steinert 1978) operationalisiert wurde, werden in der vorliegenden Untersuchung die verschiedenen Aspekte und Modalitäten von Sprache differenzierter berücksichtigt (vgl. Sprachmodell Kap. 5.3). 


\section{$5 \quad$ Das Sprachmodell}

Im vorherigen Kapitel wurde stillschweigend davon ausgegangen, dass die schulsprachliche Kompetenz ein gut definierter Begriff sei. Die genauere Analyse der wissenschaftlichen und insbesondere der (sprach-)didaktischen Literatur zeigt jedoch auf, dass weder hinsichtlich des Kompetenzbegriffs noch des Sprachbegriffs eine einheitliche Deutung vorliegt. Im Hinblick darauf, dass der Untersuchungsgegenstand des vorliegenden Forschungsvorhabens die schulsprachliche Kompetenz ist, gilt es darum, eine einheitliche Definition dieses Begriffs vorzunehmen.

\subsection{Kompetenz}

Weder im linguistischen noch im didaktisch-erziehungswissenschaftlichen Zusammenhang wird der Kompetenzbegriff einheitlich verwendet. Linguistisch hat sich im Gefolge der Diskussion um die generative Transformationsgrammatik (Chomsky 1966; 1969) eine relativ klare Gliederung zwischen den sprachgenerierenden, tiefenstrukturellen Regeln (Kompetenz) und den an der Oberfläche sich manifestierenden sprachlichen Äusserungen (Performanz) herauskristallisiert. Es wird hier - zumindest in theoretischer Hinsicht - die Sprache in zwei Modi, nämlich das potentielle sprachliche Vermögen eines "native speakers" einerseits und der effektiven Realisierung der Sprache andererseits, unterschieden. Damit wird implizit ausgesagt, dass sich aufgrund der performativen Äusserung zwar auf eine zugrundeliegende Kompetenz schliessen lässt, dass aber die Performanz nicht über das gesamte Potential sprachlicher Möglichkeiten des Sprechers Auskunft gibt. Genau diese Schwierigkeit umgeht der heute in der Sprachdidaktik und den Erziehungswissenschaften oft gebräuchliche Kompetenzbegriff. Hier verschwimmt nämlich die Trennung zwischen Kompetenz und Performanz, indem als Kompetenz sehr oft die effektive bzw. anzustrebende manifeste sprachliche Äusserung - oder genereller: die sprachliche Handlung - bezeichnet wird und nicht die potentielle "Verfügbarkeit" sprachlicher bzw. handlungsbezogener Fähigkeiten. So ist etwa Le Boterf (1994: XX) der Meinung, dass Kompetenz einzig und allein in der Manifestation, und nicht etwa in der zugrunde liegenden Disposition besteht: "La compétence ne réside pas dans les ressources (connaissances, aptitudes, ...) à mobiliser, mais dans la mobilisation même de ces ressources." Die Schwierigkeit einer solchen Definition zeigt sich, wenn die sprachliche Äusserung unter kommunikativen Gegebenheiten beleuchtet wird: Einflüsse des Kanalgeschehens, seien sie psychologischer (z. B. Motivation) oder physikalischer Natur (z. B. räumliche Bedingungen), werden hier entweder ausgeklammert oder als nicht der sprachlichen Manifestation "zugehörig" deklariert.

Was an der "Oberfläche" sprachlicher Manifestationen mobilisiert wird, ist nicht Ausdruck der gesamten sprachlichen Kompetenz, sondern wird durch situative Gegebenheiten alteriert. Es ist daher nur der beobachtbare, manifeste Ausdruck der sprachlichen Kompetenz in der aktuellen Situation. Kompetenz ist aus der Sicht des Sprachbenutzers immer mehr als Performanz. Dieser Sachverhalt zeigt sich z. B. auch in der gängigen Unterscheidung von produktivem und rezeptivem Sprachgebrauch: Die meisten Menschen manifestieren ein weit geringeres Repertoire an sprachlichen Produktionen, als sie rezeptiv zu verstehen vermögen. Es wäre trotzdem nicht angemessen, mehrere sprachliche Kompetenzkonstrukte zu postulieren, vielmehr manifestiert sich die einheitlich definierte sprachliche Kompetenz beim Schreiben und beim Lesen in unterschiedlicher Weise. Folgte man der Definition Le Boterfs, so würde die sprachliche Kompetenz in unendliche viele Teilkompetenzen zerfallen, und es wäre nach jeder Äußerung (mobilisation) die Kompetenz neu zu definieren. Das von Le Boterf aufgeworfene Problem erscheint unter diesem Blickwinkel daher eher als ein methodologisches, nämlich als jenes der Operationalisierung (d. h. des Manifestmachen) von Kompetenzen. 
Im vorliegenden Forschungsvorhaben wird von einer einheitlich definierten Kompetenz ausgegangen. Kompetenz bezeichnet das Potential eines Menschen, aufgrund seiner angeborenen und erlernten Fähigkeiten und Fertigkeiten, Probleme angemessen, d.h. situationsangepasst zu lösen. Sprachliche Kompetenz besteht im Potential eines Menschen Probleme mittels sprachlicher Handlungen zu lösen.

Damit nähern wir uns dem Kompetenzbegriff an, der auch der Harmonisierung der Fächer im Projekt HarmoS-Schweiz und insbesondere bei der Arbeit von HarmoS-Erstsprache (HarmoS-L1) zugrunde liegt. HarmoS-L1 macht eine Anleihe bei den Arbeiten von Klieme et al. (2003: 72) zur Schaffung von Bildungsstandards, welche wiederum von der Definition Weinerts (Weinert 2001: 27) beeinflusst sind: "Unter Kompetenz verstehen wir die bei Individuen verfügbaren oder von ihnen erlernten kognitiven Fähigkeiten und Fertigkeiten, bestimmte Probleme zu lösen, sowie die damit verbundenen motivationalen, volitionalen und sozialen Bereitschaften und Fähigkeiten, die Probleme in variabeln Situationen erfolgreich und verantwortungsvoll nutzen zu können."

Ob man dem zweiten Teil von Weinerts Definition folgen will und die genannten Bereitschaften in den Kompetenzbegriff einbeziehen soll, erscheint uns eher die Frage einer pädagogischen Entscheidung und weniger in einer linguistisch zu definierenden Kompetenz begründet.

\subsection{Sprachbegriff Schulsprache}

Mit dem Begriff der Schulsprache (language of education; langue de scolarisation) übernehmen wir eine Bezeichnung wie sie vom Konsortium HarmoS-Erstsprache in Anlehnung an den Sprachgebrauch der UNESCO verwendet wurde. "Mit Schulsprache bezeichnen wir jene Sprache, die als erste in der Schule verwendet wird, in der Lesen und Schreiben gelernt wird und die für einen grossen Teil der Schüler und Schülerinnen auch die erste erworbene Sprache ist" (HarmoS-Erstsprache 2007: 4). Die Schulsprache ist somit auch der Standard der lokalen Umgebungssprache in ihrer mündlichen und in ihrer schriftlichen Variante. Im gängigen Sprachgebrauch der deutschsprachigen Schweiz spricht man auch von "Hochdeutsch" und hebt damit die Schulsprache von der Mundart und damit von der örtlich gesprochenen dialektalen Umgangssprache ab. Aus linguistischer und sprachdidaktischer Sicht gilt es hier zwei Unterscheidungen zu machen:

Die erste Unterscheidung betrifft die Definition dessen, was hier als Schulsprache zu verstehen und somit die Förderaufgabe der Schule ist. Erschöpft sich die Förderung der Schulsprache im Aufbau bzw. in der Perfektionierung eines Systems von sprachlichen Regeln (Sprache als System, Sprachliche Kompetenz sensu strictu) oder beinhaltet sie auch jene situativen und adressatenbezogenen Elemente, die sprachliche Manifestationen zur Kommunikation werden lassen (Kommunikative Kompetenz, Sprachkompetenz sensu latu)?

Die zweite Unterscheidung betrifft jene der sprachlichen Modalität, die Frage nämlich, ob man Sprache unter dem Aspekt der Mündlichkeit oder jenem der Schriftlichkeit betrachten will. Beide Unterscheidungen sind für das vorliegende Projekt von Bedeutung, weil sie einerseits den Gegenstand der Untersuchung festlegen, andererseits die Gewichtung des sprachlichen Unterrichts definieren. Wir gehen kurz auf beide Unterscheidungen ein, ohne damit aber den bekannten Diskurs über den Begriff der kommunikativen Kompetenz ausführen zu wollen (Hymes 1973) ${ }^{8}$.

\footnotetext{
8 Im didaktischen Bereich fand die Diskussion unter anderem bei folgenden Autoren statt: Bünting/Kochan (1973), Klein/Wunderlich (1972), Wunderlich (1970), Wunderlich (1972).
} 


\subsubsection{Sprache als System vs. Sprache im kommunikativen Gebrauch}

Es ist vorauszuschicken, dass die Sprache im kommunikativen Gebrauch die Sprache als System umfasst, was aber umgekehrt nicht der Fall ist. M.a.W.: Der normale Sprachbenutzer verwendet in seiner natürlichen sprachlichen Kommunikation immer das sprachliche System, während dieses allein jedoch das Gelingen der Kommunikation nicht sichert.

Unter Sprache als System verstehen wir das Set von Regeln (generativer und transformativer Art), das es ermöglicht Sprache in (a) phonologischer, (b) morphologischer (c) syntaktischer und bis zu einem gewissen Grade (d) lexikalischer Sicht zu erzeugen ${ }^{9}$. Sprachliche Systemregeln erzeugen für einen "native speaker" grundsätzlich richtige Äusserungen in einer grossen Varietät. Solche Äusserungen können zudem weitgehend kontextfrei produziert, verstanden und als richtig oder falsch beurteilt werden. Grundsätzlich ist die Entwicklung dieses sprachlichen Regelsystems bei einem "native speaker" schon bei Schulbeginn abgeschlossen. Die Schule präzisiert dieses Regelsystem auf einer oberflächenstrukturellen Ebene und sorgt insbesondere für die Verschriftungsfähigkeit der sprachlichen Äusserungen. (Hauptsächlich) im schulischen Kontext werden dann auch die zusätzlichen normativen Regeln des richtigen Schreibens (Orthographie) und des richtigen Lesens (Ortholexie) erworben. Unterricht, der sich in der Hauptsache dem Erwerb des normativ richtigen Sprachgebrauchs im Sprechen, Hören, Lesen und Schreiben widmet, bezeichnen wir als systemlinguistisch orientierten Sprachunterricht. Aus einer eng linguistischen Sichtweise können richtige sprachliche Äusserungen kontextfrei richtig erzeugt und als solche beurteilt werden. Streng genommen handelt es sich um einen idealen Sprecher-Hörer, der mit niemandem kommuniziert.

\subsubsection{Sprache als Kommunikation}

Weder in der Entwicklung des einzelnen Menschen noch in der gesellschaftlichen Praxis geschieht Sprache kontextfrei. Sprache - soweit es sich nicht um angeborene Grundfunktionen handelt - wird immer in Zusammenhängen erworben, die als solche nicht rein sprachlich sind bzw. das enge Regelsystem der sprachlichen Kompetenz sensu strictu durchbrechen. Die regelhafte Korrektheit von Sprache würde der Angemessenheit kommunikativen Geschehens, d.h. der gegenseitigen Verständlichkeit kaum genügen. Weil sprachliches Verstehen fast immer Kontextwissen oder Kontextwahrnehmung impliziert, hängt Verstehen nicht einfach von sprachlichen Äusserungen im engen Sinne ab, sondern vom Einbezug des gesamten Geschehens, in dem Sprache eingebettet ist. Im kommunikativen Geschehen wird somit das unmittelbare situative Wissen (die Wahrnehmung der Situation in der Sprache geschieht), das gespeicherte Wissen und die Absichten des Sprecher/Hörers einbezogen. In der Kommunikation stellt sich somit oft nicht die Frage, ob Botschaften in korrekter Sprache verschlüsselt werden sondern, ob die Botschaft in der beabsichtigten Weise den Empfänger erreicht beziehungsweise von diesem verstanden wird.

Das Kriterium der gelungenen sprachlichen Kommunikation ist damit jenes der situativen und auf den Adressaten bezogenen Angemessenheit. In Hinblick auf das Unterrichten der Schulsprache erweitert sich damit die Zielsetzung des Sprachunterrichts. Dieser hat darauf zu achten, dass die SchülerInnen über den Erwerb der korrekten Schulsprache hinaus auch den angemessenen Umgang mit den sprachlichen Mitteln erwerben, die kommunikative Kompetenz. Es trifft zu, dass in vielen Fällen - und insbesondere im Falle der Schriftlichkeit die Kommunikation einen hohen Grad an systemlinguistischer Korrektheit erfordert. In diesem Sinne umfasst die kommunikative Kompetenz die sprachliche Kompetenz sensu

\footnotetext{
${ }^{9}$ Wir zählen aus praktischen Gründen auch die Regeln der Ortholexie und der Orthographie hier hinzu.
} 
strictu, erschöpft sich aber nicht in dieser. Die Entwicklung der kommunikativen Kompetenz bezieht sich somit sowohl auf den korrekten als auch auf den angemessenen Sprachgebrauch oder auf die Entwicklung der Fähigkeit in unterschiedlichen Situationen sprachlich angemessen zu handeln. Indem schulsprachliche Förderung das Erlernen des angemessenen Sprachhandelns anstrebt, wird sie dem Schüler aufzeigen in welchen Fällen Angemessenheit auch Korrektheit meint. Kurz: Schulsprachliche Förderung heißt immer Förderung der kommunikativen und der sprachlichen Kompetenz.

\subsection{Das Sprachmodell der Untersuchung}

Dem vorliegenden Forschungsprojekt liegt denn auch ein sprachliches Handlungsmodell zugrunde, das im Wesentlichen vom sprachlichen Pragmatismus geprägt ist. HarmoS-L1 definiert das sprachliche Handlungsmodell durch (1) die vier Modalitäten des Sprachhandelns, die umfassend gefördert werden müssen: (a) Lesen, (b) Schreiben, (c) Hören, (d) Sprechen und (2) durch fünf Aspekte des sprachlichen Handelns: (a) Sprache planen, (b) Sprache situieren, (c) Sprache reparieren, (d) Sprache realisieren, (e) Sprache evaluieren. Hinsichtlich der sprachlichen Handlungsaspekte wird rasch ersichtlich, dass diese Gliederung durch die Idee eines gesteuerten Sprachhandlungsaktes geprägt ist, der in den 80er-Jahren im Rahmen der Kognitionspsychologie entwickelt wurde (Bereiter/Scardamaglia 1987; Chun/Plass 1997; Schreiben, z. B.: Hayes/Flower 1980; Lesen, z. B.: Irwin 1986; Van Dijk/Kintsch 1983) und dann in die Sprachdidaktik (Ludwig 1983) übertragen wurde. Unter dem Gesichtspunkt der modernen Kognitionspsychologie sind diese Modelle allerdings zu rudimentär, um als Abbildung kognitiver Produktions- und Rezeptionsprozesse gelten zu können. Sie eignen sich daher auch nicht für die Erklärung psychologischer Abfolgeprozesse bei der Sprachproduktion oder -rezeption, sondern erfüllen lediglich den Zweck einer sprachdidaktisch orientierten Gliederung sprachlicher Handlungen, die sich in schulischen Kontexten als relevant erwiesen haben:

(a) Sprache planen umfasst jene Tätigkeiten, die insbesondere bei der Sprachproduktion dazu verhelfen, Texte zu gliedern, zu gewichten und in einer der Kommunikation angemessenen Form zu gestalten.

(b) Sprache situieren umfasst jene Tätigkeiten der sprachlichen Produktion bzw. Rezeption, die den Zweck oder die intendierte Wirkung der sprachlichen Kommunikation steuern bzw. erkennen lassen.

(c) Sprache reparieren umfasst alle jene Tätigkeiten der Sprache, welche zur Verbesserung von geplanten bzw. realisierten Kommunikation beitragen. Es sind dies etwa die konkreten sprachlichen Korrekturen, Rückfragen und wiederholtes Lesen von Texten sowie die metalinguistischen Kommentare, die den Inhalt- bzw. die Intention von Texten erläutern.

(d) Sprache realisieren umfasst die Umsetzung von Gedanken und Absichten in beobachtbare sprachliche Äusserungen auf der Ebene der Verbo- und Graphomotorik, des Wortes, des Satzes und des gesamten Textes, d.h. die Kodierung. In rezeptiver Hinsicht geht es um die Dekodierung von mündlich geäusserten oder geschriebenen Zeichenketten. In Erweiterung der rein verbalen Kodierung und Dekodierung werden auch textbegleitende, ikonische Repräsentationen in die Realisierung einbezogen.

(e) Sprache evaluieren umfasst die Tätigkeiten, die es ermöglichen, das kommunikative Geschehen hinsichtlich seines Inhalts, seiner Form und seines beabsichtigen oder erreichten Zwecks zu kommentieren, zusammenzufassen und zu erläutern. Die Evaluation umfasst damit auch die Beurteilung der Angemessenheit und Richtigkeit von Texten hinsichtlich ihrer Wirkung. 
Es liegt auf der Hand, dass die genannten Aspekte des kommunikativen Handelns (a) weder prozessual gedacht sind, noch dass (b) die Aspekte völlig voneinander zu trennen sind. So gibt es z. B. einen Überschneidungsbereich von Evaluieren und Situieren, was es in der Praxis manchmal erschwert, Aufgabenstellungen klar zu operationalisieren.

Andererseits erweist es sich trotz der definitorischen Schwierigkeiten als nützlicher, eine Gliederung der sprachlich-kommunikativen Handlungen in Aspekte vorzunehmen, als diese nur pauschal unter dem Gesichtspunkt sprachlicher Modalitäten zu betrachten. Unter didaktischer Betrachtungsweise lassen sich dann auch spezifische Teilkompetenzen gezielt fördern. So umfasst beispielsweise der Aspekt Situieren im Bereich des Hörens folgende Teilkompetenzen: sit 1: Die Fähigkeit, zentrale Inhalte und die kommunikative Intention einer mündlichen Äußerung zu erfassen (Wie ist die Äußerung gemeint?); sit 2: die Fähigkeit, die Funktion eines Textes (erzählend, argumentierend, informierend) zu erfassen; sit 3: die Fähigkeit, die kommunikative Absicht des Adressierenden zu erfassen; sit 4: die Fähigkeit, das eigene Hörerinteresse (Was will ich wissen?) zu definieren (gem. HarmoS-Erstsprache 2007: 21). In Abb. 2 wird das der Untersuchung zugrunde liegende Sprachmodell zusammenfassend dargestellt.

\begin{tabular}{c|c|c|c|c|c|}
\multicolumn{2}{c}{} & \multicolumn{4}{c}{ Modalitäten des sprachlichen Handelns } \\
\cline { 3 - 6 } & & \multicolumn{2}{c}{ Produktiv } & \multicolumn{2}{c}{ Rezeptiv } \\
\cline { 2 - 6 } & Situieren & Teilkompetenzen & Teilkompetenzen & Teilkompetenzen & Teilkompetenzen \\
\cline { 2 - 6 } $\begin{array}{c}\text { Aspekte des } \\
\text { Sprachhandelns }\end{array}$ & Planen & Teilkompetenzen & Teilkompetenzen & Teilkompetenzen & Teilkompetenzen \\
\cline { 2 - 6 } & Realisieren & Teilkompetenzen & Teilkompetenzen & Teilkompetenzen & Teilkompetenzen \\
\cline { 2 - 6 } & Reparieren & Teilkompetenzen & Teilkompetenzen & Teilkompetenzen & Teilkompetenzen \\
\cline { 2 - 6 } & Evaluieren & Teilkompetenzen & Teilkompetenzen & Teilkompetenzen & Teilkompetenzen \\
\hline
\end{tabular}

Abb. 2: Sprachmodell der Untersuchung

\subsection{Die Dimension: Mündlichkeit vs. Schriftlichkeit.}

Im Hinblick auf das vorliegende Forschungsvorhaben erweist es sich als notwendig, eine möglichst klare Trennung zwischen der "Schriftlichkeit" und der "Mündlichkeit" von Sprache herzustellen. Einerseits nämlich wird die mündliche Erstsprache vom Gesichtspunkt ihrer Sozialisation in andern Kontexten und in der Regel früher erworben (familiärer Kontext, Peergruppe), während die schriftliche Sprache relativ systematisch in schulischen Kontexten und damit in der Regel zu einem späteren Zeitpunkt erworben wird. Andererseits kann schriftlicher Sprachgebrauch nicht einfach als eine Übertragung von Sprechen auf Schreiben bzw. Hören auf Lesen betrachtet werden, da sich Schriftlichkeit in andern Kontexten abspielt als Mündlichkeit, und die beiden Modi der Sprache somit auch andere kognitive Strategien erforderlich machen. Feilke (2006) verweist in diesem Zusammenhang darauf, dass der Schreibprozess durch die Abwesenheit konkreter Adressaten, fehlender Lautlichkeit, der Notwendigkeit der Planung und Kohärenzerzeugung eher einem Problemlöseprozess gleichkommt, und sich von der eher habituellen Mündlichkeit grundsätzlich unterscheidet. Schreiben - im Unterschied zu Sprechen - führe zu einer tiefgreifenden Umstrukturierung des sprachlichen Wissens und erfordere dazu die entsprechende Zeit für den Kompetenzaufbau.

Tatsächlich wird in der Erstsprachdidaktik und in der Zweisprachigkeitspädagogik auf die Bedeutung der Unterscheidung Schriftlichkeit vs. Mündlichkeit aufmerksam gemacht: Cummins verweist mit seiner Unterscheidung von BICS (engl.: "conversational language") und CALP (engl.: "academic language"/Schulsprache) auf den Umstand, dass der schulische 
(Zweit-)Spracherwerb nicht einfach den Erwerb eines neuen medialen Codes bedeutet. Vielmehr handelt es sich um den Erwerb von (Bi-)Literalität, welche psychologisch betrachtet anderen Gesetzen unterliegt als die gewöhnliche Konversation des Alltags (Cummins 1979; 2004). Dieser Ansatz stimmt weitgehend mit den konstruktivistischen Ansätzen der modernen Sprachdidaktik überein: So postulieren etwa Günther (1993) und Koch-Oesterreicher (1994a) aus sprachdidaktischer Sicht zwei grundlegend unterschiedliche Konzepte von Sprachverwendung und damit auch von Sprachenlernen in Bezug auf (1) die Kommunikationsbedingungen und (2) die Versprachlichungsstrategien. Während "Konversation" (Mündlichkeit) primär face-to-face, situationsbezogen und spontan verläuft, ist Literalität (Schriftlichkeit) primär monologisch, situationsfremd, reflektiert und geplant.

Die Versprachlichung erfordert dementsprechend andere kommunikative Strategien, hinsichtlich des Strukturierungsgrad, der Verdichtung, der Abstraktion und der Hypotaxe (Günther 1993; Günther/Ludwig 1994; Koch/Oesterreicher 1994b; Olson/Torrance 1991; Ong 1987). In Abb. 3 geben wir in Anlehnung an Günther (1993) und Siebert-Ott (2001) einen vergleichenden Überblick über die Unterschiede von konzeptueller Mündlichkeit und konzeptueller Schriftlichkeit wieder. Dass der Übergang von der konzeptuellen Mündlichkeit zur konzeptuellen Schriftlichkeit in der kommunikativen Praxis nicht einem Entweder-Oder entspricht, welche sich z. B. in den verschiedenen Zwischenformen des Parlandos ausdrückt (Sieber 1998; 2000), wird hier nicht weiter diskutiert. Auf heuristischer Ebene dient das Modell jedoch dazu, Schwierigkeiten offen zu legen, mit denen insbesondere auch mehrsprachige Kinder im Bereich der Schriftlichkeit konfrontiert sind.

\begin{tabular}{|c|c|c|}
\hline & $\begin{array}{l}\text { Konzeptuelle Mündlichkeit/BICS } \\
\text { Konversationale Sprache }\end{array}$ & $\begin{array}{l}\text { Konzeptuelle Schriftlichkeit/CALP } \\
\text { Schulisch-Akademische Sprache }\end{array}$ \\
\hline $\begin{array}{l}\text { Kommunikations- } \\
\text { bedingungen }\end{array}$ & $\begin{array}{l}\text { dialogisch } \\
\text { face-to-face } \\
\text { spontan/ungeplant } \\
\text { situationsgebunden/eingebettet } \\
\text { affektoffen(-er) } \\
\text { enaktiv/ikonisch verankert } \\
\text { Nähe }\end{array}$ & $\begin{array}{l}\text { monologisch } \\
\text { räumlich und zeitlich getrennt } \\
\text { reflektiert/geplant } \\
\text { situationsentbunden/wenig eingebettet } \\
\text { affektorientiert(-er) } \\
\text { symbolisch verankert } \\
\text { Distanz }\end{array}$ \\
\hline $\begin{array}{l}\text { Versprachlichungs- } \\
\text { strategien }\end{array}$ & $\begin{array}{l}\text { prozessual } \\
\text { parataktisch } \\
\text { frei Form } \\
\text { Elaboriertheitsanspruch geringer } \\
\text { Verbindlichkeit niedrig/Vorläufigkeit }\end{array}$ & $\begin{array}{l}\text { objektivierend/vergegenständlichend } \\
\text { hypotaktisch } \\
\text { Richtigkeit/Norm } \\
\text { Elaboriertheitsanspruch höher } \\
\text { Verbindlichkeit hoch/Endgültigkeit }\end{array}$ \\
\hline Textualität & $\begin{array}{l}\text { geringe Kompaktheit } \\
\text { geringe Komplexität } \\
\text { geringe Elaboriertheit } \\
\text { geringe Strukturiertheit/Systematik }\end{array}$ & $\begin{array}{l}\text { höhere Kompaktheit } \\
\text { höhere Komplexität } \\
\text { höhere Elaboriertheit } \\
\text { höhere Strukturiertheit/Systematik }\end{array}$ \\
\hline
\end{tabular}

Abb. 3: Konzeptuelle Mündlichkeit und konzeptuelle Schriftlichkeit im Vergleich

Hinsichtlich des Schulspracherwerbs mehrsprachiger SchülerInnen stellt sich die Frage, ob die Lehrpersonen sich der Bedeutung dieser Unterscheidung bewusst seien. Besonders in dialektalen Lernumgebungen, wie dies beispielsweise in der deutschen Schweiz der Fall ist, kann der Umstand, dass mehrsprachige (und einsprachige) SchülerInnen sich mündlich relativ flüssig im hochsprachlichen Standard ausdrücken zur irrtümlichen Auffassung führen, dass auch der schreibsprachliche Standard relativ gut beherrscht würde und dass diese SchülerInnen somit keiner weiteren differenzierten Hilfen bedürften. Dies könnte zu einer erheblichen Benachteiligung der mehrsprachigen SchülerInnen führen, zumal diese im 
Bereiche der schriftlichen Sprache auch von ihren Eltern in weit geringerem Masse unterstützt werden können, als dies bei ihren einsprachigen KollegInnen der Fall ist.

\subsection{Empirische Sachverhalte zur Unterscheidung konversationale vs. schulisch akademische Sprache}

In der Regel wird in den amerikanischen Untersuchungen nach drei bis vier Jahren Schulung ein relativ konstant bleibender Unterschied in der Sprachleistung von 1 Standardabweichung zwischen bilingualen ELL (English language learner) und monolingualen englischsprachigen SchülerInnen festgestellt (Cummins 2004), sofern nicht adäquate Massnahmen bilingualer Förderung eingesetzt werden. Auch die übrigen, gross angelegten amerikanischen Vergleichsstudien berichten über ähnliche Unterschiede für die englischen Mainstream- oder Transitionsprogramme (Hakuta/Butler/Witt 2000; Hernandez-Chavez 1984; Ramirez 1992; Thomas/Collier 1997; 2002). Dieses negative Ergebnis stimmt mit der PISA-Untersuchung in den deutschsprachigen Ländern weitgehend überein. Das PISA-Konsortium Deutschland stellt z. B. bei 15-Jährigen einen Unterschied von ca. 70 Leseleistungspunkten $(1 \mathrm{SD}=100$ Punkte) zwischen zuhause nur deutsch sprechenden und zuhause nicht deutsch sprechenden Jugendlichen fest (Baumert/Schümer 2001).

$\mathrm{Zu}$ ähnlichen Ergebnissen gelangt die Schweizer PISA-Erhebung 2000. Sie berichtet über Unterschiede von 103 Punkten im Lesetest zwischen einheimischen (monolingualen) und immigrierten bilingualen Jugendlichen (Moser 2002: 119). Die Unterschiede fallen moderater aus, sobald zentrale Variablen wie beispielsweise Intelligenz, die soziale Schicht und das Geschlecht kontrolliert werden. So konnten in einer umfassenden Untersuchung bei 450 6.- bis 9.-Klässlern im Bereich des Lesens und des Schreibens Unterschiede von ca. 1 Standardabweichung beim einfachen Kohortenvergleich von bilingualen vs. monolingualen festgestellt werden. Die Unterschiede schrumpften aber beträchtlich unter Kontrolle der Intelligenz und der sozialen Schicht. Sie blieben zwischen den ethnolinguistischen Kohorten jedoch weiterhin signifikant (Müller 1997).

Ähnliche Ergebnisse erbringen die Zürcher Evaluationen bei sprachlichen Leistungen am Ende der Primarschule (Moser/Rhyn 1997; 2000): Die multiple Regression mit der abhängigen Variable "schulische Deutschleistungen" weist hochsignifikante Wirkungen der unabhängigen Variablen kognitive Leistungsfähigkeit, soziale Herkunft, Geschlechts und "Fremdsprachigkeit" auf. Die Wirkung des Migrationshintergrunds und des Geschlechts ist mit standardisierten Regressionskoeffizienten von $\beta_{\text {FREMDSPR. }}=-5.1$ und $\beta_{\text {GESCHLECHT }}=-3.8$ stark ausgeprägt. Müller (1997) stellt fest, dass sich die mehrsprachigen SchülerInnen unter der Kontrolle der zentralen exogenen Variablen bezüglich des schulsprachlichen Selbstkonzeptes/Selbstvertrauens von den einsprachigen SchülerInnen signifikant unterscheiden. Diese Variable erweist sich zusammen mit der sprachlichen Intelligenz als die zentrale Variable in der Erklärung der schulsprachlichen Leistungen sowohl vor als auch nach der Selektion in die Sekundarstufe I.

Ohne hier weitere Untersuchungen anzuführen, kann aus empirischer Sicht festgestellt werden: Die negativen Wirkungen in den Mainstream- und Transitionsprogrammen, welche die amerikanischen Evaluations- und Surveystudien (Hakuta et al. 2000; Hernandez-Chavez 1984; Ramirez 1992; Thomas/Collier 1997; 2002) beim Vergleich bilingualer Programme bezüglich der schulischen Sprachleistungen feststellen, stimmen erstaunlich genau mit jenen bei den bilingualen MigrantInnen in der Schweiz überein. Es sind dies (1) die stark unterdurchschnittlichen schulsprachlichen Leistungen mit einer Tendenz zur Stagnation auf einem relativ niedrigen Zweisprachigkeitsniveau nach ca. 4 bis 5 Jahren monolingualem Unterricht, (2) der allmähliche Verlust der Herkunftssprache bzw. die Reduktion auf den 
dialogisch umgangssprachlichen Sprachgebrauch, (3) ein vergleichsweise niedriges schulsprachliches Selbstkonzept/Selbstvertrauen und schließlich (4) ein geringerer Schulerfolg.

Auf der Ebene der mündlichen Interaktion werden kaum Mängel diagnostiziert. In der Regel sind die meisten mehrsprachigen Kinder in der Schweiz aufgewachsen und haben den Kindergarten mit ihren einsprachigen Peers durchlaufen.

Eine von uns durchgeführte Untersuchung bei 5300 Lehrlingen zeigt, dass $87.4 \%$ der bilingualen Lehrlinge als Kinder mindestens den Kindergarten in der deutschen Schweiz besucht haben und in der Regel auch die ganze Schulzeit in der Schweizer Schule verbracht haben (Müller 2005 ). Obwohl Untersuchungen zum konversationalen Sprachgebrauch von SchülerInnen in der Schweiz fehlen, kann man erfahrungsgemäss sagen, dass die meisten mehrsprachigen Kinder nicht durch eine abweichende dialektale Umgangssprache auffallen. Erst die Konfrontation mit den schulischen Anforderungen macht den Unterschied zwischen monolingualen und bilingualen SchülerInnen augenscheinlich. Während im Bereich der konversationalen Sprache sich die mehrsprachigen von den einsprachigen SchülerInnen kaum erkennbar unterscheiden, weichen sie in der schulisch - akademischen Sprache erheblich voneinander $a b$.

Hier zeigt sich eine Art Stagnation im Bereich der schulischen Sprache, die wir mit Vorsicht als eine "stagnierende schulische Lernersprache" bezeichnen. Eine aus vielen Beobachtungen hervorgehende Beurteilung von Schweizer LehrerInnen besagt, dass die schulsprachlichen Leistungen in Lesen und Schreiben bei vielen mehrsprachigen Kindern etwa auf dem Niveau von 4. bis 5. Klässlern stagnieren.

\subsection{Interimsprache/Stagnation der Lernersprache}

Im ursprünglichen theoretischen Ansatz wird die so genannte Lernersprache (Interimsprache, Interlanguage) als ein für den L2-Erwerb notwendiges, eigenständiges sprachliches Übergangssystem mit eigenen Regeln und (Lerner-)Strategien betrachtet. Diese Theorie wurde ursprünglich von Selinker (1972; Selinker 1992; Selinker, Swain/Dumas 1975) und im Gefolge dann im deutschen Sprachraum von Bausch/Kasper (1979), Knapp-Potthoff/Knapp (1982), Clahsen, Meisen/Pienemann (1983) und Pienemann (1982) formuliert. Auch die sog. "Fossilierung" der Sprache auf dem Niveau einer Lernersprache (etwa als Arbeitersprache, foreign-talk oder Pidgin) bleibt solange unproblematisch, als die kommunikative Funktion erfüllt wird und keine soziale Diskriminierung damit verbunden ist. Unter dem schulischen Aspekt bedeutet sprachliche Stagnation im Bereich der Schriftlichkeit jedoch zumeist Misserfolg und bildungsmäßiges Versagen. Wir ziehen es vor, von Stagnation anstelle von "Fossilierung der Lernersprache" zu sprechen, da in der wissenschaftlichen Literatur der Begriff der Fossilierung meist mit der Pidginisierung der mündlichen Sprechersprache verbunden ist (Übersicht bei Baker/Jones 1998: 142-150; Bartens 1996; Hellinger 1975; für die schulsprachliche Entwicklung auch McLaughlin 1985; 1987; Oksaar 2003: 112-126). Bezüglich der Kenntnisse über die Struktur und das Niveau der schulischen Interimsprachen besteht in der Schweiz jedoch ein beträchtliches Forschungsmanko, welches das vorliegende Forschungsprojekt zu beheben beabsichtigt.

\section{Forschungsanliegen und Forschungsfragen}

Das Motiv des Forschungsprojektes "Sprachliche Kompetenzen bei ein- und mehrsprachigen SchülerInnen" der Pädagogischen Hochschule Bern ist somit sprachwissenschaftlich abgestützt: Es besteht in der Schweiz ein beträchtlicher Mangel an genauen Kenntnissen über den Verlauf der schulsprachlichen Entwicklung von mehrsprachigen Kindern. Der Beitrag des Forschungsprojektes situiert sich genau hier. Wir wissen sehr viel über die selektiven Wirkungen und damit über den Bildungs(miss-)erfolg von mehrsprachigen SchülerInnen in 
der Schweiz. Wir wissen hingegen wenig über die genauen Mechanismen, die bei der Erzeugung dieses Erfolgs/Misserfolgs beteiligt sind.

Die Analyse der drei Kontexte (Kontext "Schule-Institution", "Kontext Schule-Unterricht", Kontext "Familiäre Aspiration / Zusammenarbeit Familie-Schule") soll Grundlagen für die Entwicklung von (1) diagnostischen Methoden und (2) Förderinstrumenten liefern. Dafür werden die Kontexte systematisch im Hinblick auf die Förderung mehrsprachiger SchülerInnen analysiert. Folgende Forschungsfragen werden im Projekt beantwortet:

Fragestellung 1: Analyse des Sprachstandes / Stagnation

Lassen sich in der Entwicklung schulsprachlichen Leistungen mehrsprachiger Kinder in der Schweiz Stagnationen feststellen, welche bis zum Zeitpunkt der Selektion nicht kompensierbar sind? Auf welchem schulsprachlichen Niveau bewegen sich diese Stagnationen? Wie verläuft die Erwerbskurve der schulsprachlichen Kompetenzen bei mehrsprachigen SchülerInnen im Vergleich zur Erwerbskurve der einsprachigen Kohorte? Welches sind die spezifischen Abweichungen in den Bereichen Lesen, Schreiben und Hörverstehen?

\section{Fragestellung 2: Wirkung von Kontextvariablen}

Unter welchen Bedingungen entstehen Lernverzögerungen oder allenfalls "Stagnation"? Es interessieren die:

(a) Wirkung "exogener" Bedingungen: Intelligenz, SÖS; Bildungsnähe, sprachliche und nationale Herkunft, Dauer des Schulbesuchs in $\mathrm{CH}$, Einwanderungszeitpunkt.

(b) Wirkung des schulisch-institutionellen Kontext: z. B. Berücksichtigung der speziellen Selektionsbedingungen mehrsprachiger SchülerInnen, Aspekte institutioneller Diskriminierung, Konzept der jeweiligen Institution (z. B. multikulturelle Ansätze der Schule).

(c) Wirkung des schulisch-unterrichtlich Kontexts: Niveaueinschätzungen durch LehrerInnen; didaktische Interventionen durch LehrerInnen, Differenzierungsgrad des Unterrichts hinsichtlich mehrsprachiger SchülerInnen.

(d) Familiäre Aspiration / Zusammenarbeit Schule-Elternhaus: z. B. Unterstützungsmöglichkeiten in der Familie bei schulischen Aufgaben

\section{Fragestellung 3: Unterscheidung von Sprachebenen}

Ist es möglich, dass die Stagnation bei der Entwicklung von schulisch-standardsprachlichen Kompetenzen und der damit verbundene (schul-)sprachliche Misserfolg durch die falsche Einschätzung tatsächlicher sprachlicher Fähigkeiten nicht nur nicht erkannt, sondern zum Nachteil des/r mehrsprachigen Schülers/in sogar noch befördert wird? Wir vermuten, dass folgende Faktoren zum schulischen Versagen der mehrsprachigen SchülerInnen beitragen:

(a) Mangelnde Unterscheidung zwischen der konzeptuellen Schriftlichkeit und der konzeptuellen Mündlichkeit bzw. der konversationalen Sprache von der schulischkognitiven Sprache durch die Lehrpersonen.

(b) Reduktion der Aspekte der konzeptuellen Schriftlichkeit auf normative Aspekte der Schreibsprache oder eher technisch-wahrnehmerische Aspekte beim Lesen.

Fragestellung 4: Mangelnde Berücksichtigung der spezifischen sprachlichen Realität des Elternhauses durch die Schule und LehrerInnen

Beachten Lehrpersonen bei der Delegierung von schulsprachlichen Lernaufgaben ans Elternhaus in ausreichendem Masse, ob die Eltern auch über einen ausreichenden Grad an Literalität verfügen, um ihre Kinder zu unterstützen? Wie wirkt sich die Delegierung von unterrichtlichen Bildungsinhalten, aus dem Bereich der Schulsprache (Schreiben und Lesen) 
ans Elternhaus, auf den Bildungserfolg mehrsprachiger Kinder aus? In welchem Masse vermag das Elternhaus solche Aufgaben überhaupt wahrzunehmen? Folgende Aspekte werden spezifisch betrachtet:

(a) Der Einfluss des Literalitätsgrades im Elternhaus

(b) Die Zusammenarbeit zwischen der Schule/den Lehrpersonen und der Familie.

\section{$7 \quad$ Methode}

Die sprachlichen Kompetenzen werden auf zwei Ebenen erhoben:

(1) HarmoS-L1-Test national: Es werden die gesamtschweizerisch (Sprachen D, F, I) validierten Testverfahren verwendet. Diese Testverfahren messen die fünf Sprachhandlungsaspekte (Planen, Situieren, Realisieren, Evaluieren, Reparieren) in vier Sprachmodalitäten (Schreiben, Lesen, Sprechen, Hören).

(2) Die formalen Sprachkompetenzen in den Bereichen Lesen und Schreiben werden mittels der drei standardisierten Sprachtests ELFE (Lenhard/Schneider 2006), Salzburger Lesescreening (Auer et al. 2005; Mayringer/Wimmer 2003) und HSP (May 2002) erhoben.

Die Erhebung sowohl formaler Sprachkompetenzen als auch pragmatisch-funktionaler Sprachkompetenzen ermöglicht es, in umfassender Weise den schulsprachlichen Entwicklungsverlauf ein- und mehrsprachiger SchülerInnen in einer Quasi-LängsschnittStudie zu erheben. Es handelt sich um eine quantitative Untersuchung auf drei Altersstufen (2./3. Klasse, 5./6. Klasse und 8./9. Klasse) und mit zwei Messzeitpunkten im Abstand von einem Jahr. Die Stichprobe umfasst 543 einsprachige und 583 mehrsprachige SchülerInnen (59 Schulkassen) aus dem Kanton Zürich. Die geplanten Erhebungen beinhalten nebst der Messung der Sprachkompetenz auch Erhebungen (Fragbogen und Interview) zu den schulischen Kontexten: Schule als Institution (Fragebogen und Interview mit der Schulleitung), Unterricht (Lehrpersonenbefragung) und Familie (Elternfragebogen). Erste Ergebnisse zum hier vorgestellten Projekt können Ende 2008 berichtet werden.

\section{$8 \quad$ Bedeutsamkeit der geplanten Studie}

\subsection{Wissenschaftliche Bedeutung}

\subsubsection{Erziehungswissenschaftlicher Beitrag/Bildungsstandards}

Jede Entwicklung von zu erfüllenden Bildungsstandards trägt die Gefahr in sich, dass bestimmte soziale Gruppen aus der Betrachtungsweise ausgeblendet werden, weil die curricular $\mathrm{zu}$ formulierenden Zielnormen sich an majoritären Bevölkerungskohorten orientieren. Werden Bildungsstandards primär zu Selektionszwecken benutzt, so kann sich die Einführung von Bildungsstandards für mehrsprachige SchülerInnen negativ auswirken, da ihre Sprache unter dem Aspekt einer defizitären Ausstattung betrachtet wird. Es ist daher von grosser Wichtigkeit, Zielnormen zu schaffen, welche die spezifischen Bedürfnisse benachteiligter Bildungsgruppen beachten.

Der diesbezüglich in der Schweiz herrschende Mangel an Informationen über die Mechanismen, die zum schulischen Erfolg/Misserfolg bei mehrsprachigen Schülerinnen führen, soll durch die vorliegende Untersuchung behoben werden. Indem drei grundlegende schulische Kontexte im Hinblick auf die Förderung mehrsprachiger SchülerInnen analysiert und Sprachentwicklungsdaten systematisch erhoben werden, hilft die vorliegende Studie, 
Bildungsstandards zu entwickeln, die der Förderung auch heterogener SchülerInnengruppen dienen.

\subsubsection{Forschungsmethodischer Beitrag: Schaffung von Grundlagen für Evaluations- instrumente}

Eine wesentliche Herausforderung der vorliegenden Untersuchung besteht in der Entwicklung von Evaluationsinstrumenten, welche die Merkmale "innovativer" und "migrationsfreundlicher" schulischer Kontexte erfassen. Als erster Schritt dazu wurde bereits eine Delphi-ExpertInnenbefragung durchgeführt (Müller/Rösselet 2007), die Grundlagen für eine systematische Erfassung schulischer Kontexte liefert.

\subsubsection{Psychologisch-linguistischer Beitrag: Erkenntnisse über den Sprachstand von mehrsprachigen SchülerInnen im Vergleich zur Zielnorm}

Weder für monolinguale noch bilinguale SchülerInnen bestehen in der Schweiz Forschungsergebnisse, welche (a) anhand von Zielnormen im Sinne von einheitlichen Bildungsstandards und (b) unter Berücksichtigung genau definierter schulischer Zweisprachigkeitskontexte erhoben wurden. Indem die vorliegende Untersuchung mit Griff auf die schulsprachlichen Bildungsstandards und den dafür entwickelten Testaufgaben durchgeführt wird, werden erstmals genaue Vergleiche zwischen der Zielnorm der Gesamtgruppe der Jahrgangskohorten und der Teilkohorte mehrsprachiger SchülerInnen möglich. Die Ergebnisse geben zudem genauen Aufschluss über den Entwicklungsverlauf der Schulsprache in klar definierten Bereichen.

\subsection{Soziale und erzieherische Bedeutung}

\subsubsection{Bildungsplanung/Förderpädagogischer Ansatz}

Die genaue Analyse der schulsprachlichen Kompetenzen mehrsprachiger SchülerInnen im Vergleich zur Zielnorm ermöglicht es, im Bereich der Bildungsplanung, eine genaue Stufung der Kompetenzansprüche für mehrsprachige SchülerInnen vorzunehmen. Dies entspricht den Ansprüchen der Förderpädagogik, die - im Unterschied zu einem selektiven Ansatz - von klar gestuften und individuell ausgerichteten Bildungszielen ausgeht, um die SchülerInnen schrittweise an den "Standard" heranzuführen.

\subsubsection{Didaktisch-erzieherische Aspekte}

Schließlich erhalten wir aufgrund der Kontextanalyse Daten über die notwendige Ausstattung von Schulen, welche den gesetzlich und pädagogisch geforderten Ansprüchen der Integration von mehrsprachigen MigrantInnen entsprechen. Die Daten beziehen sich auf schulischinstitutionelle und didaktische Massnahmen und zeigen auch auf, welchen Ansprüchen Familien mehrsprachiger Kinder in Bezug auf die Literalität zu genügen vermögen und wo die Schule ihrerseits Stütz- und Fördergefässe anbieten muss.

\subsection{Wirtschaftliche Bedeutung}

Nicht unterschätzt werden sollte auch die wirtschaftliche Bedeutung der Studie. Analysen in der Schweiz (Wolter/Weber 1998; 1999a; 1999b) und im Ausland (Alsalam/Conley 1995; Psacharopoulos 1987; 1994) bestätigen die ökonomischen Vorteile der Mehrsprachigkeit. Das überdurchschnittliche Bildungsversagen mehrsprachiger Menschen in der Schweiz und die damit verbundene schulische und berufliche Allozierung in Ausbildungsgänge mit niedrigen Anforderungsniveaus führen - unter bildungsökonomischen Gesichtspunkten - zu einer generell mangelnden Wertschöpfung des potentiell vorhandenen Bildungskapitals. 


\section{Literaturangaben}

Alsalam, Nabeel/Conley, Ron (1995): "The Rate of Return to Education. A Proposal for an Indicator". In: OECD (ed.): Education and Employment. Paris: 83-109.

Auer, Michaela et al. (2005): SLS 5-8. Salzburger Lese-Screening für die Klassenstufen 5-8. Göttingen/Bern/Wien.

Baker, Colin/Jones, Sylvia Prys (1998): Encyclopedia of Bilingualism and Bilingual Education. Clevedon Hall.

Bandura, Albert (1977): "Self-Efficacy. Toward a Unifying Theory of Behavioral Change". Psychological Review 84: 191-215.

Bandura, Albert (1986): Social Foundations of Thought and Action. A Social Cognitive Theory. Englewood Cliffs.

Bandura, Albert (1997): Self-Efficacy. The Exercise of Control. New York.

Bartens, Angela (1996): Der kreolische Raum. Geschichte und Gegenwart. Helsinki.

Baumert, Jürgen/Schümer, Gundel (2001): "Familiäre Lebensverhältnisse, Bildungsbeteiligung und Kompetenzerwerb". In: PISA-Konsortium, Deutsches (ed.): PISA 2000. Basiskompetenzen von Schülerinnen und Schüler im nationalen Vergleich. Opladen.

Bausch, Karl-Richard/Kasper, Gabriele (1979): "Der Zweitsprachenerwerb. Möglichkeiten und Grenzen der 'grossen' Hypothesen". Linguistische Berichte 64: 3-36.

Bereiter, Carl/Scardamaglia, Marlene (1987): The Psychology of Written Composition. Hillsdale N.J.

Bünting, Karl-Dieter/Kochan, Detlef C. (1973): Linguistik und Deutschunterricht. Kronberg.

Chomsky, Noam (1966): Cartesian linguistics. A chapter in the history of rationalist thought. New York.

Chomsky, Noam (1969): Aspekte der Syntaxtheorie. Frankfurt.

Chun, Dorothy, M./Plass, Jan, L. (1997): "Research on Text Comprehension in Multimedia Environments". Language Learning \& Technology 1/1: 60-81.

Clahsen, Harald/Meisel, Jürgen/Pienemann, Manfred (1983): Deutsch als Zweitsprache. Der Spracherwerb ausländischer Arbeiter. Tübingen.

Clément, Richard (1980): "Ethnicity, Contact and Communicative Competence in a Second Language". In: Giles, Howard/Robinson, William P. /Smith, Philip M. (eds.): Language. Social Psychological Perspectives. Oxford: 147-154.

Corradi Vellacott, Maja/Wolter, Stefan, C. (2002): "Soziale Herkunft und Chancengleichheit". In: BfS (ed.): Für das Leben gerüstet? Die Grundkompetenzen der Jugendlichen Nationaler Bericht der Erhebung PISA 2000. Neuchâtel.

Cummins, Jim (1979): "Linguistic Interdependence and the Educational Development of Bilingual Children". Review of Educational Research, 49/2: 222-251.

Cummins, Jim (2004): Language, Power an Pedagogy. Bilingual Children in the Crossfire. Clevedon.

Feilke, Helmuth (2006): "Literalität. Kultur, Handlung, Sttruktur". In: Panagiotopoulou, Argyro/Wintermeyer, Minika (eds.): Schriftlichkeit. Interdisziplinär. Voraussetzungen, Hindernisse und Fördermöglichkeiten. Frankfurt a. M.: 13-30. (= Frankfurter Beiträge zur Erziehungswissenschaft 11).

Gardner, Robert C. (1979): "Social Psychology Aspects of Second Language Acquisition". In: Giles, Howard/St. Clair, Robert (eds.): Language and Social Psychology Oxford: 193-220.

Gardner, Robert C. (1985): Social Psychology and Second Language Learning. The Role of Attitudes and Motivation. London.

Gardner, Robert C./Lalonde, Richard N./Pierson, R. (1983): "The Socio-Educational Model of Second Language Acquistion. An Investigation Using Lisrel Causal Modeling". Journal of Language and Social Psychology 2/1: 1-15. 
Gardner, Robert C./Moorcroft, R./Metforda, J. (1989): "Second Language Learning in Immersion Programme. Factors Influencing Acquistion and Retention". Journal of Language and Social Psychology 8/5: 287-305.

Giles, Howard/Byrne, Jane (1982): "An Intergroup Approach to Second Language Acquisition". Journal of Multilingual and Multicultural Development 1: 17-40.

Günther, Hartmut (1993): "Erziehung zur Schriftlichkeit". In: Eisenberg, Peter/Klotz, Peter (eds.): Sprache gebrauchen. Sprachwissen erwerben. Stuttgart: 85-95.

Günther, Hartmut/Ludwig, Otto (Eds.) (1994): Schrift und Schriftlichkeit. Ein interdisziplinäres Handbuch internationaler Forschung. Berlin.

Häberlin, Urs/Imdorf, Christian/Kronig, Winfried (2004): Von der Schule in die Berufslehre. Untersuchungen zur Benachteiligung von ausländischen und von weiblichen Jugendlichen bei der Stellensuche. Bern/Stutgart/Wien.

Hakuta, Kenji/Butler, Yuko Goto/Witt, Daria (2000): How Long Does It Take to English Learners to Attain Proficiency? Santa Barbara, CA.

HarmoS-Erstsprache (2007): Schritte zu einem Kompetenzmodell Erstsprache. EDKKonsortium HarmoS-Erstsprache. Zürich.

Hayes, John R./Flower, Linda S. (1980): "Identifying the organization of writing processes". In: Gregg, Lee W. /Steinberg, Erwin R. (eds.): Cognitive Processes in Writing. Hillsdale: 3-30.

Hellinger, Marlies (1975): Englisch-orientierte Pidgin- und Kreolsprachen. Entstehung, Geschichte und sprachlicher Wandel. Darmstadt.

Hernandez-Chavez, Eduardo (1984): The Inadequacy of English Immersion Education as a Approach for Language Minority Students. Sacramento C.A.

Hutmacher, Walo (1995): "Enfant d'ouvriers ou enfants d'immigrés?" In: Poglia, Edo et al. (eds.): Pluralité culturelle et éducation en Suisse. Etre migrant II. Bern etc.: 107-136.

Hymes, Dell (1973): "Über linguistische Theorie, kommunikative Kompetenz und die Erziehung unterpriviligierter Kinder." In: Kochan, Detlef C. (ed.): Sprache und kommunikative Kompetenz. Stuttgart: 109-130.

Imdorf, Christian (2001): "Von der Schulbank in die Berufswelt". Vierteljahrschrift für Heilpädagogik und ihre Nachbargebiete 70/3: 256-267.

Imdorf, Christian (2004): Schulqualifikation und Berufsfindung. Wie Geschlecht und nationale Herkunft den Übergang in die Berufsbildung strukturieren. Wiesbaden.

Irwin, Judith Westphal (1986): Teaching Reading Comprehension Processes. New Jersey.

Klein, Wolfgang/Wunderlich, Dieter (eds.) (1972): Aspekte der Soziolinguistik. Frankfurt a. M.

Klieme, Eckhard (2003): Zur Entwicklung nationaler Bildungsstandards. Expertise. Bonn: Bundesministerium für Bildung und Forschung.

Knapp-Potthoff, Annelie/Knapp, Karlfried (1982): Fremdsprachenlernen und -lehren. Stuttgart.

Koch, Peter/Oesterreicher, Wulf (1994): "Schriftlichkeit und Sprache". In: Günther, Hartmut/Ludwig, Otto (eds.): Schrift und Schriftlichkeit. Ein interdisziplinäres Handbuch internationaler Forschung. 1. Halbband. Berlin: 587-604.

Kronig, Winfried (2003): Flawed Selection. The Example of Immigrant Children in Swiss Education Systems. Jyväskylä.

Kronig, Winfried/Haeberlin, Urs/Eckhard, Michael (2000): Immigrantenkinder und schulische Selektion. Pädagogische Visionen, theoretische Erklärungen und empirische Untersuchungen zur Wirkung integrierender und separierender Schulformen in den Grundschuljahren. Bern/Stuttgart/Wien.

Lalonde, Richard N./Gardner, Robert C. (1984): "Investigating a Causal Model of Second Language Acquisition. Where Does Personality Fit?" Canadian Journal of Behavioural Science 15/3: 224-237. 
Lambert, Wallace E. (1974): "Culture and Language as Factor in Learning and Education". In: Aboud, Frances E. /Meade, Robert D. (eds.): Cultural Factors in Learning and Education. Bellingham/Washington: 55-83.

Lambert, Wallace E. (1979): "Language as a Factor of Intergroup Relationships". In: Giles, Howard/St. Clair, Robert (eds.): Language and Social Psychology. Oxfod: 186-192.

Lambert, Wallace E. (1981): "Bilingualism and Language Acquisition". In: Winitz, Harris (ed.): Native Language and Foreign Language Acquisition. New York.

Le Boterf, Guy (1994): De la compétence. Essai sur un attracteur étrange. Paris.

Lenhard, Wolfgang/Schneider, Wolfgang (2006): ELFE 1-6. Ein Leseverständnistest für Erst- bis Sechstklässler. Göttingen/Bern/Wien.

Bundesamt für Statistik (BfS) (1997) (ed.): Integration: (k)eine Erfolgsgeschichte: ausländische Kinder und Jugendliche im schweizerischen Bildungssystem. Bern: Bundesamt für Statistik

Ludwig, Otto (1983): "Einige Gedanken zu einer Theorie des Schreibens". In: Grosse, Siegfried (ed.): Schriftsprachlichkeit. Düsseldorf: 37-73.

May, Peter (2002): Hamburger Schreib-Probe HSP. 6. aktualisierte und erweiterte Aufl. Göttingen.

Mayringer, Heinz/Wimmer, Heinz (2003): SLS 1-4. Salzburger Lese-Screening für die Klassenstufen 1-4. Göttingen/Bern/Wien.

McLaughlin, Barry (1985): Second Language Acquisition in Childhood. Vol. 1. Hillsdale N.J. McLaughlin, Barry (1987): Theories in Second Language Learning. London.

Meyer, Thomas (2004): Wie weiter nach der Schule? Zwischenergebnisse des Jugendlängsschnitts. Bern.

Meyer, Thomas/Stalder, Barabara E./Matter, Monika (2003): Bildungswunsch und Wirklichkeit. Thematischer Bericht der Erhebung PISA. Neuchâtel.

Moser, Urs (2002): "Kulturelle Vielfalt in der Schule. Herausforderung und Chance". In: Schweizerische Konferenz der kantonalen ErziehungsdirektorInnen (EDK), Bundesamt für Statistik (BfS) (eds.): Bildungsmonitoring Schweiz. Für das Leben gerüstet? Die Grundkompetenzen der Jugendlichen. Nationaler Bericht der Erhebung PISA 2000. Neuchâtel: 113-135.

Moser, Urs/Rhyn, Heinz (1997): Evaluation der Sekundarstufe I im Kanton Zürich. Bedingungen des Lernerfolgs. Zweiter Bericht. Zürich.

Moser, Urs/Rhyn, Heinz (2000): Lernerfolg in der Primarschule. Eine Evaluation der Leistungen am Ende der Primarschule. Aarau.

Müller, Romano (1993): Sozialpsychologische Grundlagen des schulischen Zweitspracherwerbs. Eine empirische Studie bei zweisprachigen und einsprachigen SchülerInnen aus der 6.-10. Klasse in der Schweiz. Inauguraldissertation an der Phil-hist. Fak. der Uni Bern. Universität Bern.

Müller, Romano (1996): "Sozialpsychologische Variablen des schulischen Zweitspracherwerbs von Migrantenkinder". In: Schneider, Hans Jakob /Hollenweger, Judith (eds.): Mehrsprachigkeit und Fremdsprachigkeit. Arbeit für die Sonderpädagogik Luzern: 33-89.

Müller, Romano (1997): Sozialpsychologische Grundlagen des schulischen Zweitspracherwerbs bei MigrantenschülerInnen. Theoretische Grundlagen und empirische Studien bei zweisprachigen und einsprachigen SchülerInnen aus der 6.-10. Klasse in der Schweiz. Vol. 21. Aarau/Frankfurt a. M./Salzburg.

Müller, Romano (2000): "Über den Monolingualismus der Schweizer Schulen und seine Folgen für die mehrsprachigen SchülerInnen". Babylonia. A Journal of Language Teaching and Learning 1/8: 74-80. 
Müller, Romano (2001): "Die Situation der ausländischen Jugendlichen auf der Sekundarstufe II in der Schweizer Schule. Integration oder Benachteiligung". Schweizerische Zeitschrift für Bildungswissenschaften 23/2: 265-298.

Müller, Romano (2005): Berufswahl und Lehre. Berufliche Orientierungs- und Entscheidungsprozesse ausländischer und schweizerischer Jugendlicher im Vergleich. Bern.

Müller, Romano (2005): Von der Sekundarstufe I zur Sekundarstufe II. Schweizerische und ausländische Jugendliche im Übergang zur Lehre und zum Gymnasium. Vortrag anlässlich der Berner Studien- und Kongresswoche "Heterogenität und Integration". Bern.

Müller, Romano (2006 $)$ : Berufswahl und Lehre. Berufliche Orientierungs- und Entscheidungsprozesse ausländischer und schweizerischer Jugendlicher im Vergleich. Schlussbericht zuhanden der Forschungskommission der Pädagogischen Hochschule Bern. Forschungsprojekt Nr. 0002w05. Bern.

Müller, Romano (2006 $)$ : "Von der Sekundarstufe I zur Sekundarstufe II. Schweizerische und ausländische Jugendliche im Übergang zur Lehre und zum Gymnasium". In: Tanner, Albert et al. (eds.): Heterogenität und Integration. Umgang mit Ungleichheit und Differenz Zürich: 143-174.

Müller, Romano/Rösselet, Stephan (2007): "Interventions destinées au perfectionnement des compétences linguistiques en L2 (code standard-scolaire) des élèves migrants plurilingues selon le jugement d'expert(e)s. Un sondage représentatif basé sur la méthode d'interrogation d'expert(e)s Delphi". Vortrag anlässlich des Colloque International de Didactique Cognitive. Université de Toulouse. 19-20-21 septembre 2007. Toulouse.

Oksaar, Els (2003): Zweitsprachenerwerb. Stuttgart.

Olson, David R./Torrance, Nancy (eds.) (1991): Orality and Literacy. Cambridge.

Ong, Walter J. (1987): Oralität und Literalität. Die Technologisierung des Wortes. Opladen.

Pienemann, Manfred (1982): "Untersuchung zur Synchronisierung von natürlichem und gesteuertem Zweitspracherwerb. Projektbeschreibung". Deutsch Lernen 7/1: 81-87.

Psacharopoulos, George (1987): "The Cost-Benefit-Model". In: Psacharopoulos, George (ed.): Economics of Education. Research and Studies Oxford: 342-347.

Psacharopoulos, George (1994): "Returns on Investment in Education. A Global Update." World Development 22/9: 1325-1343.

Ramirez, J. David (1992): "Executive Summary". Bilingual Research Journal 16: 1-62.

Rüesch, Peter (Ed.). (1998): Spielt die Schule eine Rolle? Schulische Bedingungen ungleicher Bildungschancen von Immigrantenkindern. Eine Mehrebenenanalyse. Bern.

Selinker, Larry (1972): "Interlanguage". International Review of Applied Linguistics10: 209231.

Selinker, Larry (1992): Rediscovering Interlanguage. London/New York.

Selinker, Larry/Swain, Merill/Dumas, Guy (1975): "The Interlanguage Hypothesis Extended to Children". Language Learning 25: 139-152.

Sieber, Peter (1998): Parlando in Texten. Zur Veränderung kommunikativer Grundmuster in der Schriftlichkeit. Tübingen. (= Reihe Germanistische Linguistik).

Sieber, Peter (2000): "Parlando in Texten. Zur Veränderung kommunikativer Grundmuster in der Schriftlichkeit." Zeitschrift für Dialektologie und Linguistik 68:102-104.

Siebert-Ott, Gesa (2001): Zweisprachigkeit und Schulerfolg. Die Wirksamkeit von schulischen Modellen zur Förderung von Kindern aus zugewanderten Sprachminderheiten. Ergebnisse der Schulforschung. Bönen.

Steinert, Joachim (1978): Allgemeiner Deutscher Sprachtest (ADST). Göttingen.

Tajfel, Henri (1978): Differentiation between Social Groups. London.

Tajfel, Henri/Turner, John (1979): "An Integrative Theory of Intergroup Conflict". In: Austin, William G. /Worchel, Stephen (eds.): The Social Psychology of Intergroup Relations. Monterey: 33-47. 
Thomas, Wayne P./Collier, Virginia P. (1997): School Effectiveness for Language Minority Students. Washington, DC. www.ncbe.gwu.edu.

Thomas, Wayne P./Collier, Virginia P. (2002): A National Study of School Effectiveness for Language Minority Student's Long-Term Academic Achievement. Santa Cruz.

Van Dijk, Teun A./Kintsch, Walter (1983): Strategies of discourse comprehension. New York.

Weinert, Franz E. (2001): "Vergleichende Leistungsmessung in Schulen. Eine umstrittene Selbstverständlichkeit". In: Weinert, Franz E. (ed.), Leistungsmessungen in Schulen. Weinheim/Basel: 17-31.

Wolter, Stefan C./Weber, Bernhard A. (1998): Rates of Return to Education. Continental Europe's Dilemma. Vortrag anlässlich des $62^{\text {nd }}$ Annual Meeting of the Midwestern Economics Association (18-21 März 1998), Chicago.

Wolter, Stefan C./Weber, Bernhard A. $\left(1999^{\mathrm{a}}\right)$ : "On the Measurement of Private Raters of Return to Education." Jahrbücher für Nationalökonomie und Statistik 218/5\&6: 607-618.

Wolter, Stefan C./Weber, Bernhard A. $\left(1999^{b}\right)$ : "Skilling the Unskilled. A Question of Incentives?" International Journal of Manpower 20/3/4: 254-269.

Wunderlich, Dieter (1970): "Die Rolle der Pragmatik in der Linguistik". Der Deutschunterricht 22/4: 5-41.

Wunderlich, Dieter (1972): "Begriffszusammenhang innerhalb der Soziolinguistik". In: Engel, Ulrich/Schwenke, Olaf (eds.): Gegenwartssprache und Gesellschaft Düsseldorf: 64-70. 